\title{
Viscoelastic Properties of Dumbbell-Shaped Polystyrenes in Bulk and Solution.
}

Yuya Doi, ${ }^{* \dagger, *}$ Atsushi Takano, ${ }^{* \dagger}{ }^{\dagger}$ Yoshiaki Takahashi, ${ }^{*}{ }^{\S}$ and Yushu Matsushita ${ }^{\dagger, / /}$

${ }^{*}$ Nagoya University, ${ }^{*}$ Kyushu University and "Toyota Physical and Chemical Research Institute

\section{S1. Preparation and characterization of dumbbell-shaped PS samples.}

In this study, two dumbbell-shaped polystyrene (PS) samples, D-30/120/30 and D-30/240/30, were newly prepared in the same manner as reported previously. ${ }^{\mathrm{S} 1}$ Here, the results of preparation for D-30/240/30 are explained while adequately displaying the chromatograms in the process of preparation. First, Figure S1 shows SEC profiles of a coupling reaction process between R-30 $\left(M_{\mathrm{w}}=33.7 \mathrm{~kg} / \mathrm{mol}, M_{\mathrm{w}} / M_{\mathrm{n}}=1.02\right.$, purity $\left.=99.9 \%\right)$ and $\mathrm{L}-240\left(M_{\mathrm{w}}=241 \mathrm{~kg} / \mathrm{mol}, M_{\mathrm{w}} / M_{\mathrm{n}}=1.03\right) . \quad$ In a sealed glass apparatus under high vacuum, R-30 $(3.0 \mathrm{~g}, 9.0 \times 10$ ${ }^{-5} \mathrm{~mol}$; Figure S1a) and bifunctional living L-240 (0.8 g, $3.3 \times 10^{-6} \mathrm{~mol}$; Figure S1b) were reacted, and a coupling product (3.8 g, Figure S1c) was obtained. In Figure S1c, in addition to the peak of excess molar amount of unreacted R-30 (eluted at 20-22 min), bimodal peaks appeared at shorter elution time (12-14 min).

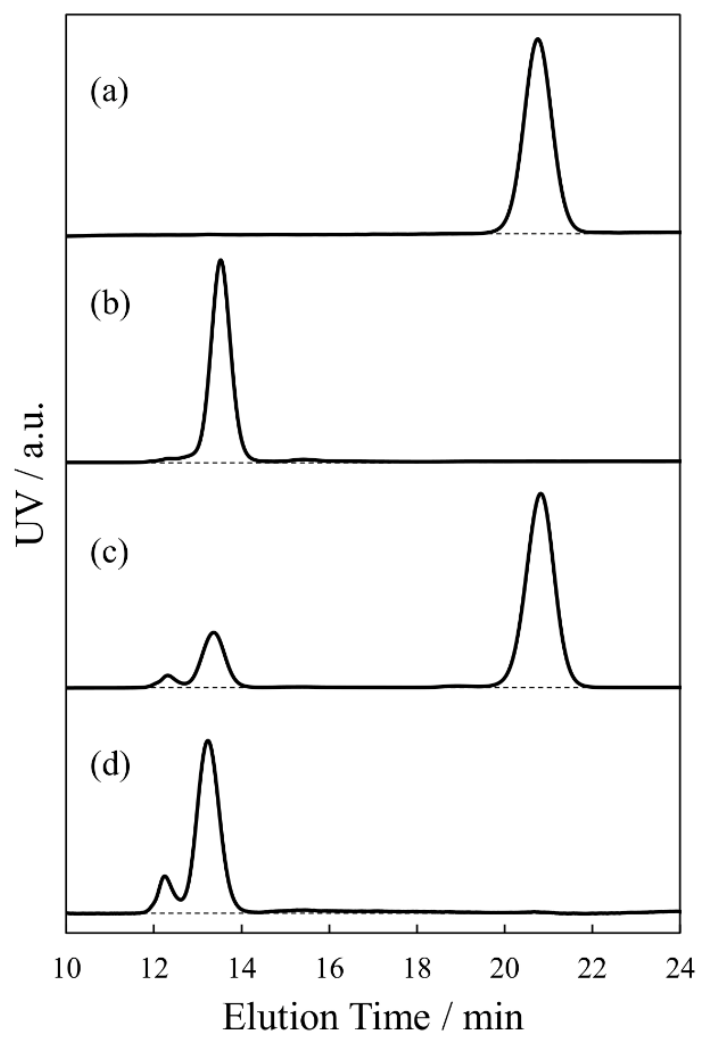

Figure S1. SEC profiles of (a) R-30, (b) L-240, (c) a coupling product between R-30 and L-240, and (d) a product after excluding unreacted R-30. The measurements were conducted in THF by using SEC apparatus reported previously ${ }^{\mathrm{S} 1, \mathrm{~S} 2}$ and two KW-804 (Shodex) columns. 
To confirm the creation of the targeted dumbbell polymer in the coupling product, Figure S2 shows the SEC profile of the product with the $M_{\mathrm{w}}$ trace detected by a multi-angle light scattering (MALS) detector, compared with that for L-240. Note that two SEC columns were used in Figure S1, while four columns were used in Figure S2 to achieve high resolution of the peaks. Thus, the elution time in Figure S2 is almost twice longer than that in Figure S1. We can find two things in Figure S2 that (i) the elution time of the main peak of the coupling product (25-28 min) is clearly shorter than that of L-240 and (ii) the $M_{\mathrm{w}}$ trace of the coupling product is evidently higher especially at shorter elution time and has a wider distribution than L-240. These facts suggest that the targeted dumbbell chain, D-30/240/30, is included in the single peak (25-28 min) together with unreacted L-240 and singletail tadpole S-30/240. Moreover, the peaks eluted at much shorter time (23-25 min) are thought to be a twin-tail tadpole and higher $M_{\mathrm{w}}$ polycondensations. ${ }^{\mathrm{S} 1}$

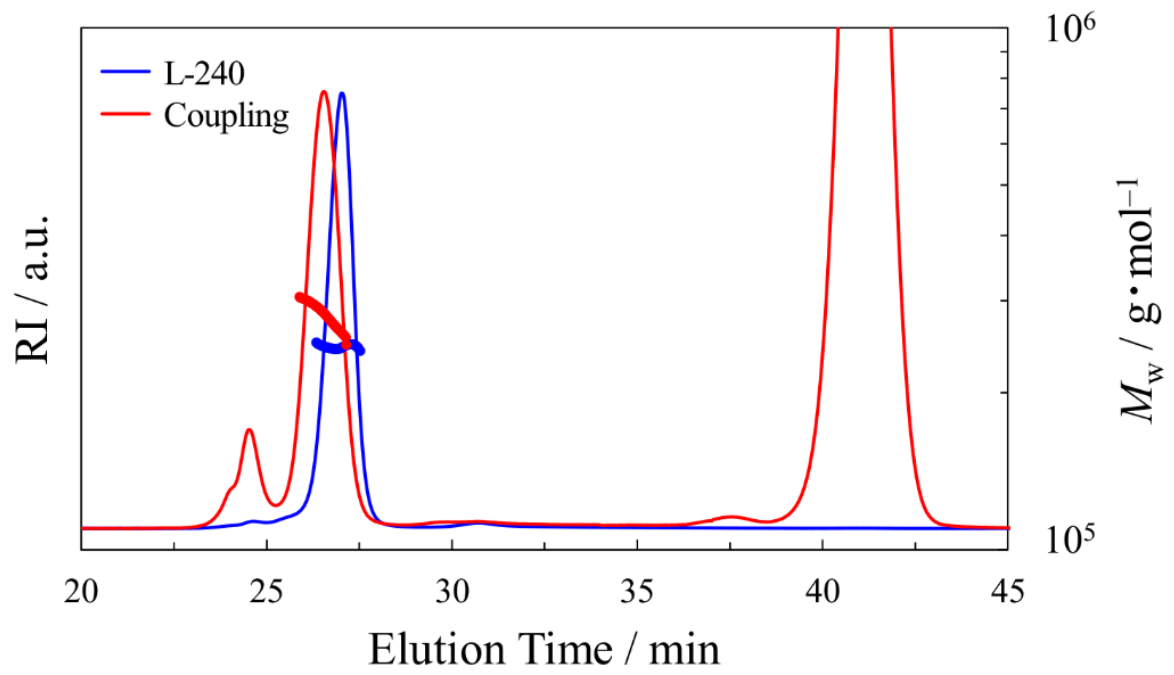

Figure S2. SEC profile of a coupling product between R-30 and L-240 (red; corresponds to the sample in Figure S1c), compared with that of L-240 (blue). The bold curves indicate $M_{\mathrm{w}}$ traces detected by a MALS detector (on right axis). The measurements were conducted by using four KW-804 (Shodex) columns to attain high resolution.

To prepare a highly-purified dumbbell-shaped polymer, multistep SEC and interaction chromatography (IC) fractionations were carried out. First, a coupling product was treated by preparative SEC fractionation to exclude unreacted R-30, and the result is shown in Figure S1d. It is clear that most of the unreacted R-30 was successfully excluded, and the yield is ca. $900 \mathrm{mg}$. Subsequently, IC measurement of the product was conducted, and the result is shown in Figure S3a. In the same manner as reported previously, ${ }^{S 1}$ three main peaks indicated by sold arrows appeared in IC mode, and they are conceived to be linear, single-tail and dumbbell chains in order from the shorter elution time. Note that the peaks eluted at 10-20 min correspond to solvent. To isolate the targeted dumbbell polymer, IC fractionation was performed. The yield of the product after the fractionation is ca. 250 
$\mathrm{mg}$, but the product includes a certain amount of impurities such as single-tail and twin-tail polymers other than the targeted dumbbell one (no SEC and IC profiles are shown here). Thus, further SEC and IC fractionations were performed to the sample, and finally the highly-purified dumbbell PS, D-30/240/30 was obtained as shown in Figure S3b (IC) and S3d (SEC). The yield of the final product is ca. $100 \mathrm{mg}$. It is clear from the IC profile in Figure S3b that linear and single-tail polymers eluted shorter than the dumbbell one were completely excluded after the repeated fractionations. In addition, the SEC profile in Figure S3d exhibits a unimodal narrow peak compared with that before fractionation in Figure S3c.

We have made no explanation here, but the other dumbbell PS sample, D-30/120/30, was also successfully prepared (ca. $70 \mathrm{mg}$ ) in the same manner as D-30/240/30.
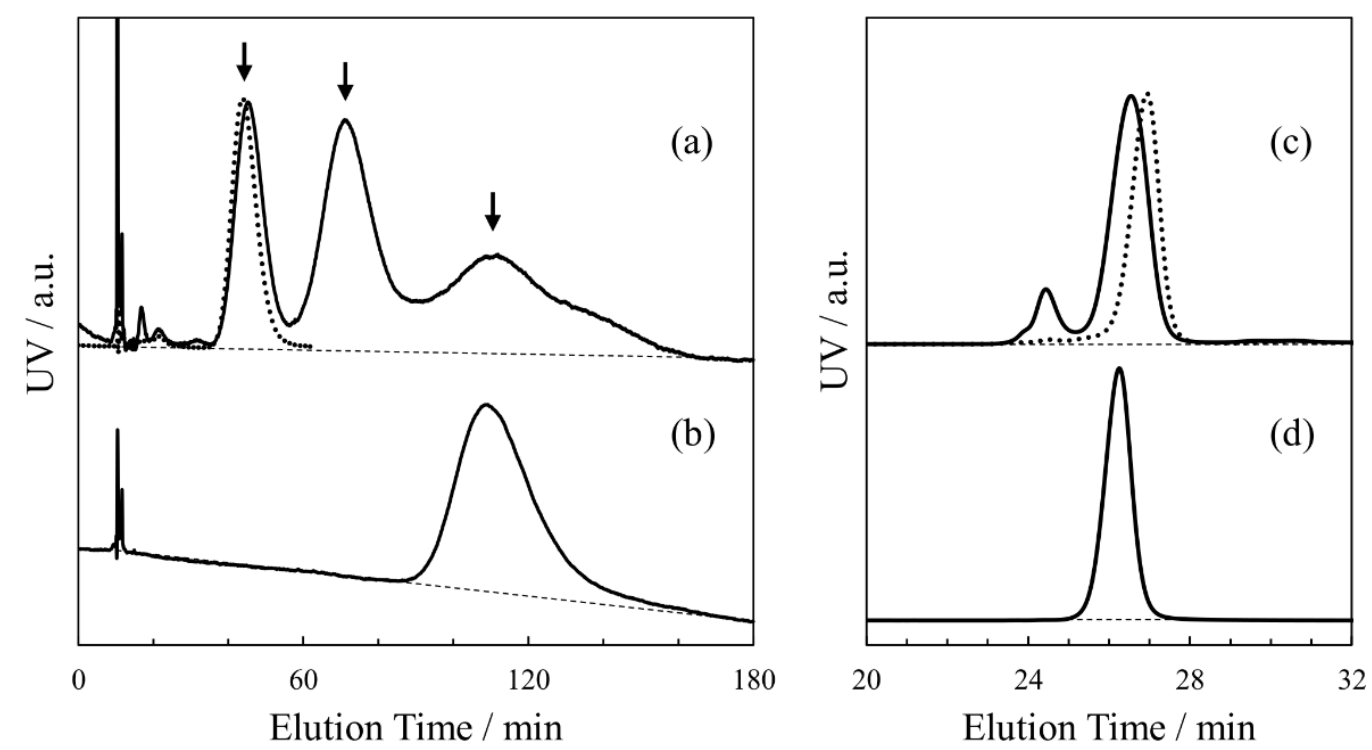

Figure S3. IC profiles of (a) a product after excluding R-30 and (b) an isolated D-30/240/30 after repeated SEC and IC fractionations. SEC profiles of (c) and (d) correspond to the samples shown in (a) and (b), respectively. Dotted curves in panels (a) and (c) are the profiles of L-240. The condition of IC measurements is the same with those reported previously, ${ }^{\mathrm{S} 1}$ and the elution behavior is controlled by changing the column temperature. Three arrows in panel (a) indicate the peaks corresponding to linear, single-tail and dumbbell polymers in order of elution time.

\section{S2. Characterization of dumbbell-shaped PS samples after rheological measurements.}

After dynamic viscoelastic and stress relaxation measurements of the dumbbell PS samples, SEC and IC measurements were conducted to check whether chain degradations occurred. Figure S4a and S4b show SEC profiles of D-30/240/30 before and after rheological measurements, respectively, while Figure S4c and S4d show their corresponding IC profiles. In Figure S4b, extremely small peak shoulders (less than $1 \%$ ) newly appeared on both sides of the main peak probably due to thermal degradation and crosslinking, but essentially the main peak 
keeps its shape even after rheological measurements. The situation is the same for IC measurements as shown in Figure S4c and S4d, i.e., D-30/240/30 keeps its peak shape and no new peaks were detected. Thus, we can safely say that very little chain degradation/crosslinking was occurred for D-30/240/30, and it was scarcely influenced on the result of the rheological measurements. The results are essentially the same for D-30/120/30, although its SEC and IC profiles were not shown here.
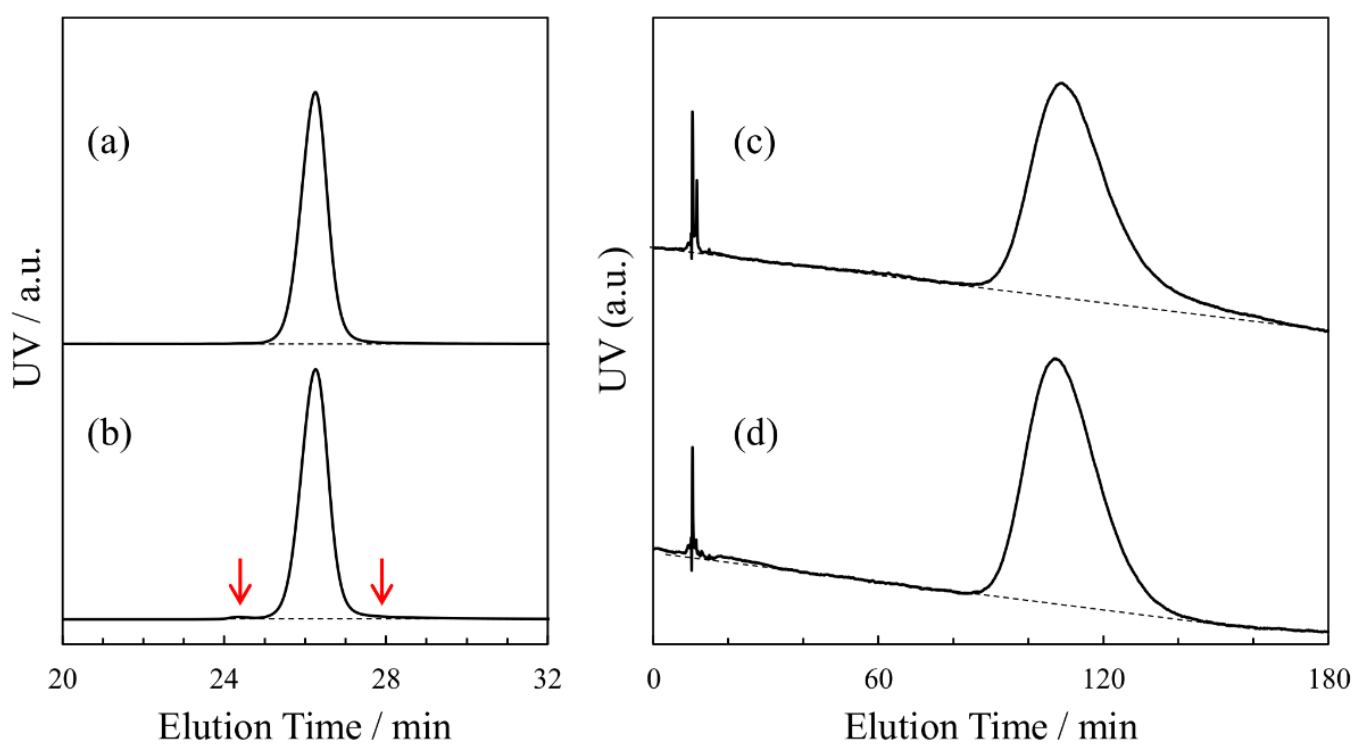

Figure S4. SEC profiles of D-30/240/30 (a) before and (b) after rheological measurements, and their IC profiles in (c) and (d). The red arrows in (b) indicate where the peak shoulders newly appeared after rheological measurements.

\section{S3. Details of rheological data for dumbbell-shaped PS samples in bulk.}

\section{S3.1. Dynamic oscillatory measurements}

As described in the main text, the original dynamic moduli data of two dumbbell PS samples in the glasstransition region at high $\omega\left(>10^{4} \mathrm{~s}^{-1}\right)$ are considerably lower than those for the other linear and ring samples. The original master curves of $G^{\prime}, G^{\prime \prime}$ and $\tan \delta$ for two dumbbell samples without arbitrary vertical shift are shown in Figure S5 and S6. In contrast to the moduli data, $\tan \delta$ at high $\omega$ side shows good agreement between the dumbbell and linear PS samples. In addition, the discussion on the temperature dependence of $a_{\mathrm{T}}$ in the main text revealed that the averaged local friction for the dumbbell chains is basically the same with linear PS samples. Thus, we conceive that the lower moduli for the dumbbells are originated from an experimental artifact where the samples did not completely cover/attach the geometry to some extent during the measurements. Based on the above facts, we made a vertical shift of the moduli for dumbbell polymers arbitrarily to agree with those for linear ones at high $\omega$ regime $\left(>10^{4} \mathrm{~s}^{-1}\right)$, i.e., $A=0.20$ for $\mathrm{D}-30 / 120 / 30$ and 0.22 for $\mathrm{D}-30 / 240 / 30$ in a logarithmic scale. 

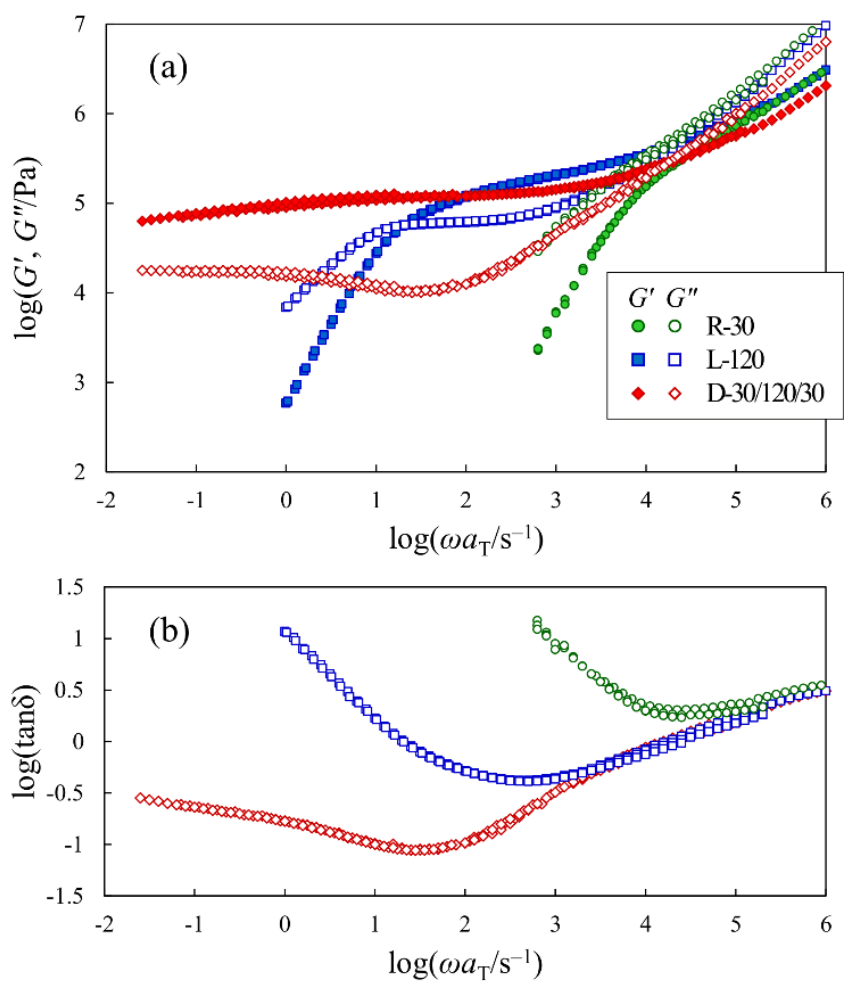

Figure S5. Angular frequency $\omega$ dependence of (a) $G^{\prime}$ and $G^{\prime \prime}$, and (b) $\tan \delta$ for D-30/120/30 (without any arbitrary vertical shift) and its components, L-120 and R-30. The data at each $T$ for the samples are reduced at $180{ }^{\circ} \mathrm{C}$.
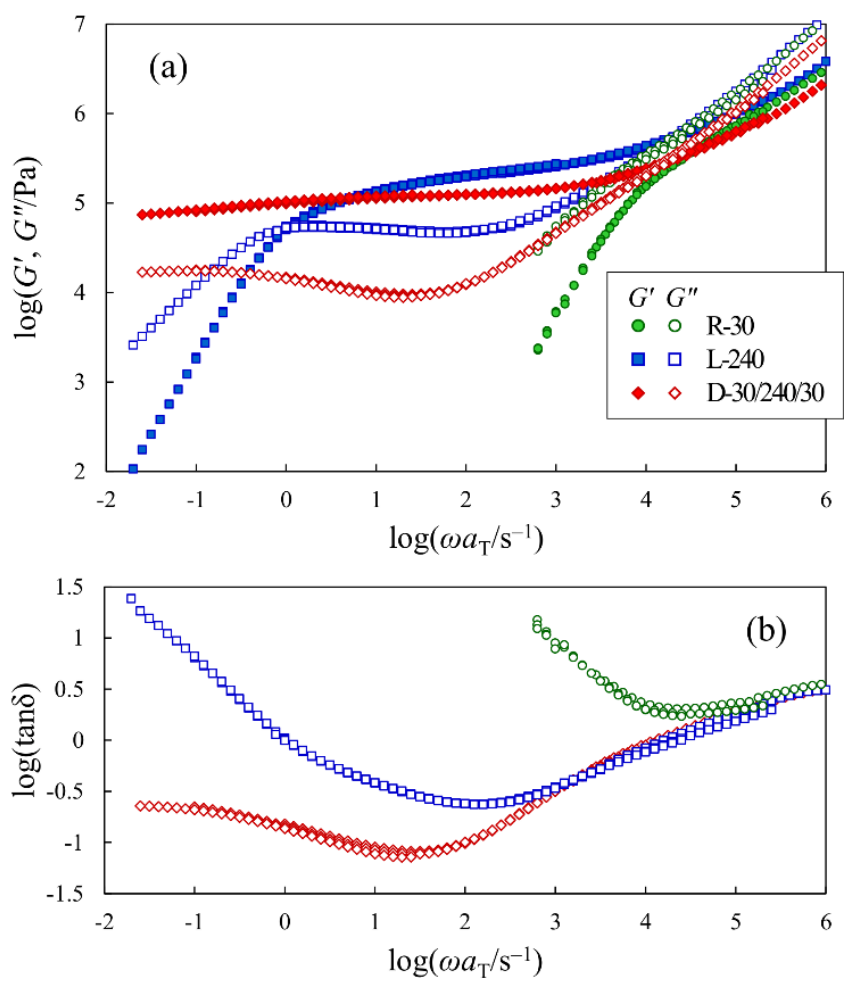

Figure S6. Angular frequency $\omega$ dependence of (a) $G^{\prime}$ and $G^{\prime \prime}$, and (b) $\tan \delta$ for D-30/240/30 (without any arbitrary vertical shift) and its components, L-240 and R-30. The data at each $T$ for the samples are reduced at $180{ }^{\circ} \mathrm{C}$. 
In Figure S7, master curves of $G^{\prime}, G^{\prime \prime}$ and $\tan \delta$ for two dumbbell PS samples, D-30/120/30 and D-30/240/30, are directly compared in the same panel. In Figure S7, $G^{*}(\omega)$ for two dumbbells are almost completely overlapped with each other. When we carefully compare the data, a small difference is observed in $G^{\prime \prime}$ as well as $\tan \delta$ at $\omega=10^{-1} \sim 10^{2} \mathrm{~s}^{-1}$. This difference might originate from the difference of the chain motion of two dumbbells where both ring parts are tethered by the intermolecular threading.
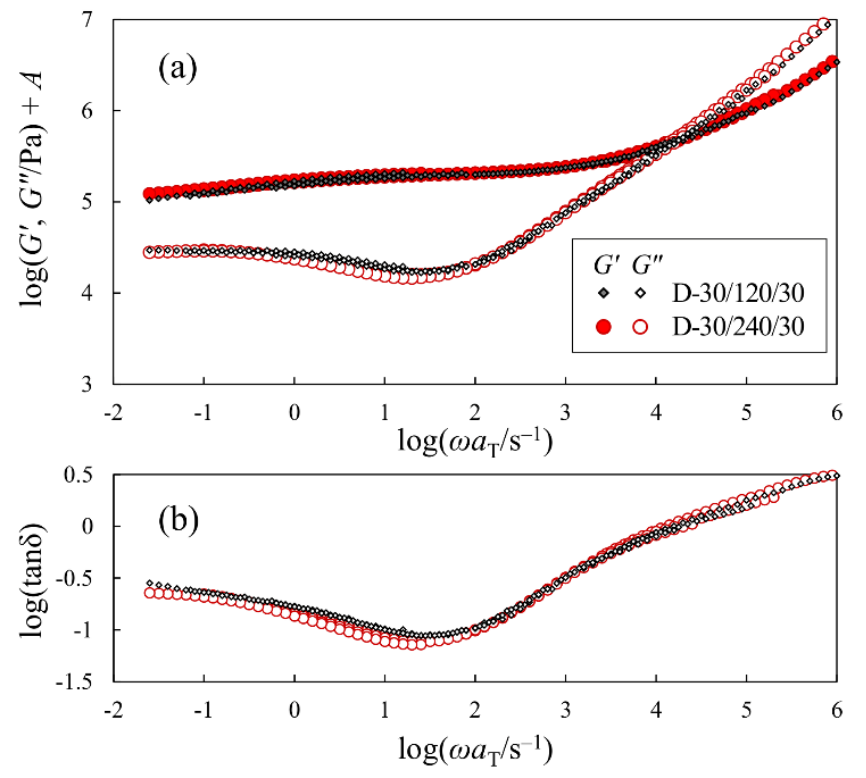

Figure S7. Comparison of (a) $G^{\prime}$ and $G^{\prime \prime}$ and (b) $\tan \delta$ for two dumbbell PS samples, D-30/120/30 (black) and D$30 / 240 / 30$ (red), reduced at $180^{\circ} \mathrm{C}$. The moduli for D-30/120/30 and D-30/240/30 are vertically shifted with $A=0.20$ and 0.22 , respectively, in a logarithmic scale.

\section{S3.2. Stress relaxation measurements}

Figure S8 show the $G(t)$ data of two dumbbell PS samples, D-30/120/30 and D-30/240/30, compared with those of linear PS ones with various molecular weights at $180{ }^{\circ} \mathrm{C}$.

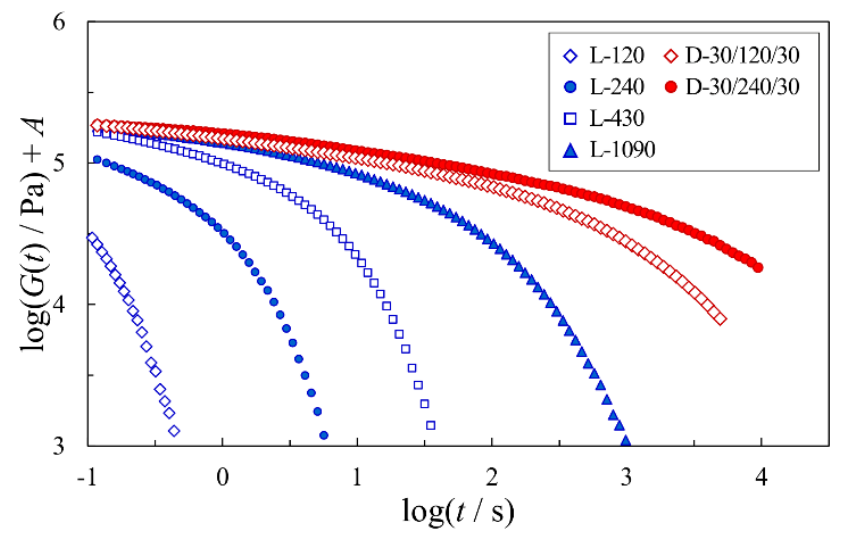

Figure S8. $G(t)$ of two dumbbell PS samples, D-30/120/30 and D-30/240/30, compared with those of four linear PS ones with different $M_{\mathrm{w}}$ at $180{ }^{\circ} \mathrm{C}$. 
Figure S9 compares the $G^{*}(\omega)$ obtained from dynamic oscillatory measurements with those obtained by transforming $G(t)$ from stress relaxation measurements for two dumbbell PS samples and their component linear ones, reduced at $180{ }^{\circ} \mathrm{C}$. To transform $G(t)$ data to $G^{*}(\omega)$, we performed a multimode analysis by fitting the $G(t)$ data with a sum of exponentially decaying modes, $\sum_{p} g_{p} \exp \left(-t / \tau_{p}\right)$, from long to short $t$ by following the method called as Procedure X. ${ }^{\mathrm{S} 3}$

We confirmed that the $G^{*}(\omega)$ data transformed from $G(t)$ merged with the $G^{*}(\omega)$ from oscillatory measurements in some digits of $\omega$, i.e., $10^{-2} \leq \omega / \mathrm{s}^{-1} \leq 10^{0}$. Note that the deviation of $G^{\prime \prime}(\omega)$ at $10^{0} \leq \omega / \mathrm{s}^{-1} \leq 10^{1}$ calculation from $G(t)$ originated from the limitation of $G(t)$ data at short $t$ side. However, this deviation hardly affects the estimation of $G^{*}(\omega)$ at lower $\omega\left(\leq 10^{0} \mathrm{~s}^{-1}\right)$. Thus, in this study, we can equivalently treat the $G^{*}(\omega)$ data from oscillatory measurements and the $G(t)$ data from stress relaxation measurements.
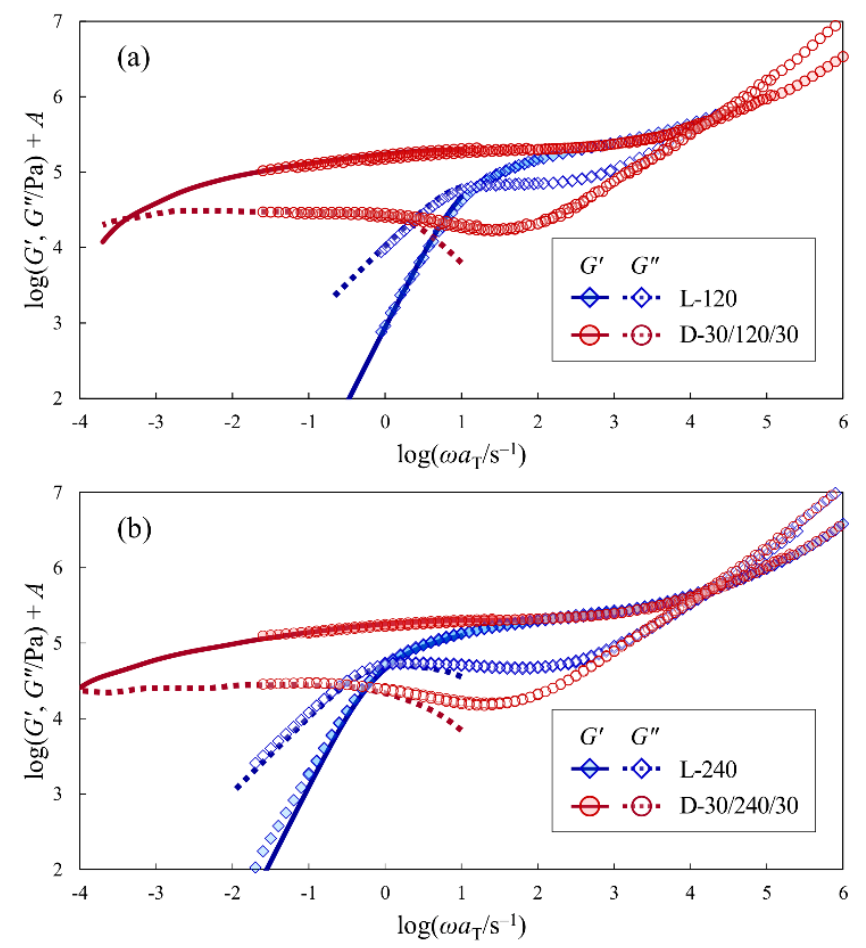

Figure S9. Comparison of $G^{\prime}$ and $G^{\prime \prime}$ obtained from dynamic oscillatory measurements (symbols) and those transformed from $G(t)$ in stress relaxation measurements (solid and dotted curves) for (a) D-30/120/30 and (b) D-30/240/30, reduced at $180^{\circ} \mathrm{C}$. The moduli for the corresponding linear components, L-120 and L-240, are also shown in panel (a) and (b), respectively. The moduli for D-30/120/30 and D-30/240/30 are vertically shifted with $A=0.20$ and 0.22 , respectively, in a logarithmic scale. 


\section{S4. Details of rheological data for dumbbell-shaped PS samples in solutions.}

The overlap concentrations $c^{*}$ of the polymer solution samples measured in this study are estimated. Using the radius of gyration $R_{\mathrm{g}}$ in solution, $c^{*}$ is defined as:

$$
c^{*}=3 M_{\mathrm{w}} / 4 \pi R_{\mathrm{g}}{ }^{3} N_{\mathrm{A}}
$$

where $M_{\mathrm{w}}$ is the molecular weight and $N_{\mathrm{A}}$ is the Avogadro's number. In this study, we basically discuss the rheological behavior of polymer solutions in $\theta$-solvent. The dimension of linear PS chain in $\theta$-solvent (i.e., cyclohexane at $35^{\circ} \mathrm{C}$ ) is known as: $:^{\mathrm{S}, \mathrm{S} 5}$

$$
R_{\mathrm{g}}=0.0279 \times M_{\mathrm{w}}{ }^{1 / 2}
$$

For three linear PS solutions (L-240, L-430 and L-1090) used in this study, their $R_{\mathrm{g}}$ and $c^{*}$ values are estimated and summarized in Table S1. Here, we basically regard that the $R_{\mathrm{g}}-M_{\mathrm{w}}$ relationship for linear PS in cyclohexane at $35{ }^{\circ} \mathrm{C}$ in eq. $\mathrm{S} 2$ is still valid in our experimental systems, i.e., in dioctyl phthalate (DOP) at $22{ }^{\circ} \mathrm{C}$. For the dumbbell-shaped PS sample, D-30/240/30, its chain dimension in $\theta$-solvent is not experimentally determined. Just as a reference, here we make a rough estimation of $R_{\mathrm{g}}$ and $c^{*}$ by using the relative molecular weight of D$30 / 240 / 30\left(M_{\mathrm{w}, \mathrm{rel}}=263 \mathrm{~kg} / \mathrm{mol}\right)$ determined by SEC with standard linear PS samples. It is reasonable that $M_{\mathrm{w}, \text { rel }}$ is somewhat lower than the absolute $M_{\mathrm{w}}(=314 \mathrm{~kg} / \mathrm{mol})$ determined by SEC-MALS because the dumbbell D30/240/30 should possess slightly smaller chain dimensions, namely, slightly longer elution time in SEC, than the supposed linear PS sample (L-314) having the same absolute $M_{\mathrm{w}}$ with D-30/240/30. In fact, the elution time in SEC directly reflects the size (to be accurate, hydrodynamic radius) of a polymer chain in dilute solution. Thus, using the relative molecular weight for D-30/240/30 is reasonable to estimate $c^{*}$ of D-30/240/30.

Table S1. $R_{\mathrm{g}}$ and $c^{*}$ for dumbbell-shaped and linear PS solutions in DOP at $22{ }^{\circ} \mathrm{C}$

\begin{tabular}{ccc}
\hline Samples & $R_{\mathrm{g}} / \mathrm{nm}^{\mathrm{a}}$ & $c^{*} / \mathrm{g} \cdot \mathrm{mL}^{-1}$ \\
\hline L-240 & 13.3 & 0.041 \\
L-430 & 17.6 & 0.031 \\
L-1090 & 28.2 & 0.019 \\
D-30/240/30 & $14.3^{\mathrm{b}}$ & $0.036^{\mathrm{b}}$
\end{tabular}

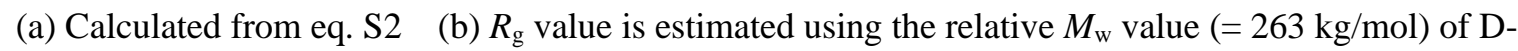
30/240/30 determined by SEC with standard linear PS samples

The information, i.e., concentrations $c$ (in $\mathrm{g} / \mathrm{mL}$ ) and zero-shear viscosities $\eta_{0}$, for all dumbbell-shaped PS (D30/240/30) and linear PS (L-240, L-430 and L-1090) solutions in dioctyl phthalate (DOP) at $22^{\circ} \mathrm{C}$ is summarized 
in Table S2. As explained in the main text, $\eta_{0}$ of the solutions is estimated from the relationship as $\eta_{0}=$ $\lim _{\omega \rightarrow 0}\left\{G^{\prime \prime}(\omega) / \omega\right\}$

Table S2. Information for dumbbell-shaped and linear PS solutions in DOP at $22{ }^{\circ} \mathrm{C}$

\begin{tabular}{ccccccccc}
\hline & \multicolumn{2}{c}{$\mathrm{D}-30 / 240 / 30$} & \multicolumn{2}{c}{$\mathrm{L}-240$} & \multicolumn{2}{c}{$\mathrm{L}-430$} & \multicolumn{2}{c}{$\mathrm{L}-1090$} \\
$\mathrm{wt} \%$ & $c / \mathrm{g} \cdot \mathrm{mL}^{-1}$ & $\eta_{0} / \mathrm{Pa} \cdot \mathrm{s}$ & $c / \mathrm{g} \cdot \mathrm{mL}^{-1}$ & $\eta_{0} / \mathrm{Pa} \cdot \mathrm{s}$ & $c / \mathrm{g} \cdot \mathrm{mL}^{-1}$ & $\eta_{0} / \mathrm{Pa} \cdot \mathrm{s}$ & $c / \mathrm{g} \cdot \mathrm{mL}^{-1}$ & $\eta_{0} / \mathrm{Pa} \cdot \mathrm{s}$ \\
\hline 30 & $0.27_{5}$ & 12600 & $0.26_{7}$ & 1970 & $0.25_{2}$ & 6840 & - & - \\
20 & $0.18_{9}$ & 368 & $0.19_{0}$ & 94.5 & $0.17_{7}$ & 499 & $0.15_{3}$ & 10400 \\
12 & $0.11_{6}$ & 8.44 & $0.12_{1}$ & 14.0 & $0.10_{8}$ & 36.8 & $0.09_{3}$ & 491 \\
8 & $0.07_{3}$ & 1.15 & $0.07_{3}$ & 1.96 & $0.07_{6}$ & 6.10 & $0.05_{6}$ & 20.6 \\
6 & $0.05_{8}$ & 0.49 & $0.05_{3}$ & 0.84 & $0.06_{0}$ & 1.87 & $0.04_{9}$ & 13.3 \\
3 & $0.02_{9}$ & 0.16 & $0.03_{5}$ & 0.29 & $0.02_{8}$ & 0.33 & $0.02_{2}$ & 0.73 \\
\hline
\end{tabular}


Figure S10 show the $\omega$ dependence of $G^{\prime \prime} / \omega$ for D-30/240/30 and two linear PS (L-240 and L-430) solutions in DOP at $22{ }^{\circ} \mathrm{C}$ at relatively low $c(8,6$ and $3 \mathrm{wt} \%)$.
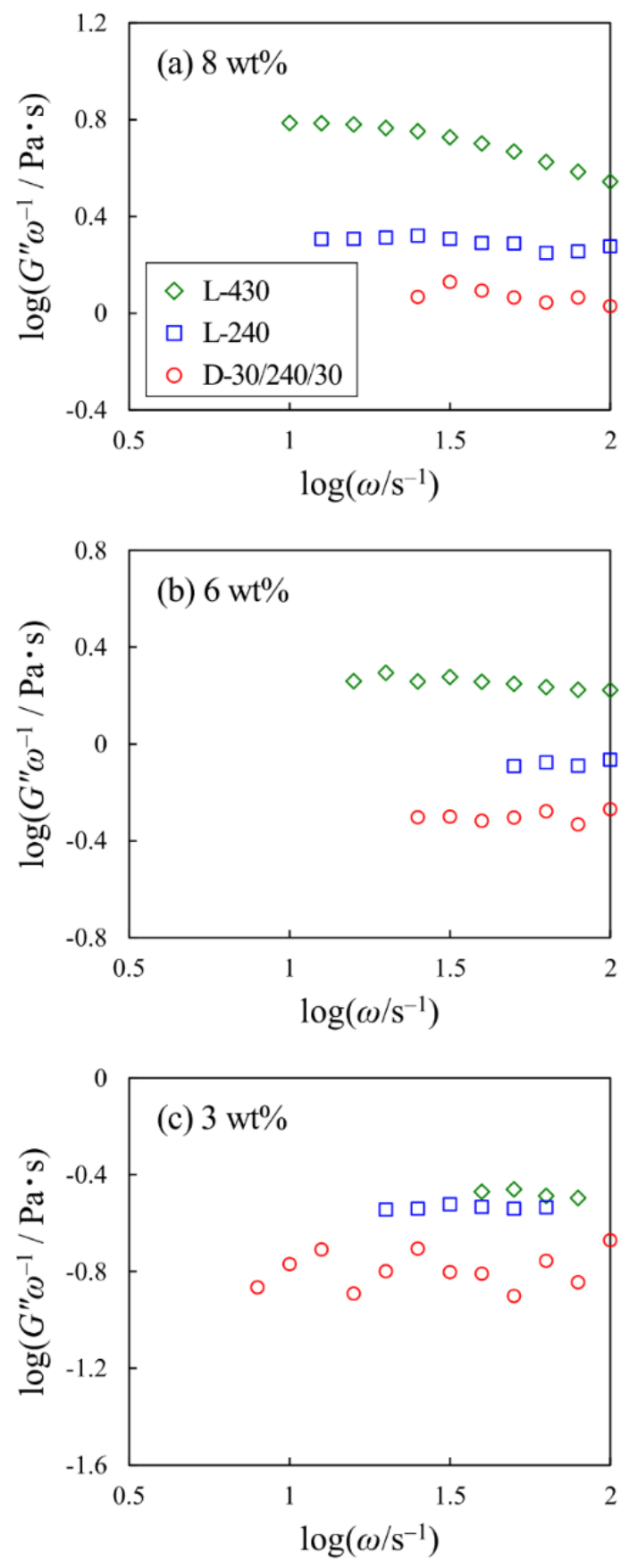

Figure S10. $G^{\prime \prime} / \omega$ vs $\omega$ for D-30/240/30 solutions in DOP at (a) 8, (b) 6 and (c) 3 wt $\%$ at $22^{\circ} \mathrm{C}$, compared with those for linear PS, L-240 and L-430, solutions. 
Figure S11a and S11b show the $\omega$ dependence of $G^{*}$ and $G^{\prime \prime} / \omega$, respectively, for L-1090 solutions at various $c$ in DOP reduced at $22{ }^{\circ} \mathrm{C}$.
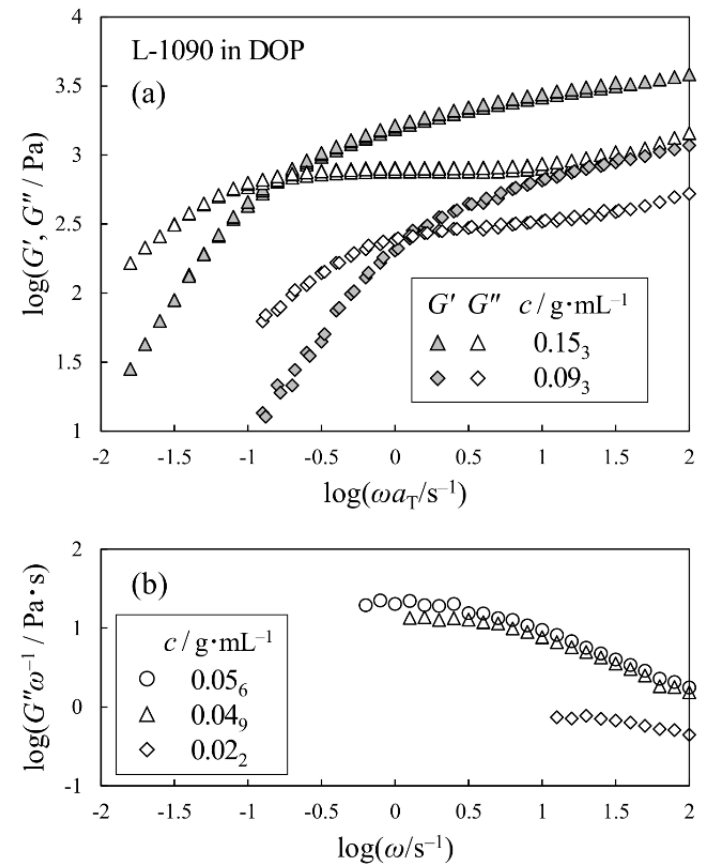

Figure S11. (a) $G^{\prime}$ and $G^{\prime \prime}$ vs $\omega$ and (b) $G^{\prime \prime} / \omega$ vs $\omega$ for L-1090 solutions in DOP at different $c$ reduced at $22^{\circ} \mathrm{C}$.

Table S3 summarizes the steady-state recoverable $J_{\mathrm{e}}$ values for D-30/240/30 and linear PS solutions in DOP. For the lowest $c(3 \mathrm{wt} \%)$, the compliances were not accurately estimated due to the low values of $G^{\prime}$.

Table S3. $c$ and $J_{\mathrm{e}}$ for dumbbell-shaped and linear PS solutions in DOP at $22{ }^{\circ} \mathrm{C}$

\begin{tabular}{|c|c|c|c|c|c|c|c|c|}
\hline \multirow[b]{2}{*}{ wt $\%$} & \multicolumn{2}{|c|}{ D-30/240/30 } & \multicolumn{2}{|c|}{ L-240 } & \multicolumn{2}{|c|}{ L-430 } & \multicolumn{2}{|c|}{ L-1090 } \\
\hline & $\begin{array}{c}c \\
\mathrm{~g} \cdot \mathrm{mL}^{-1}\end{array}$ & $\begin{array}{l}10^{3} J_{\mathrm{e}} \\
\mathrm{Pa}^{-1} \\
\end{array}$ & $\begin{array}{c}c \\
\mathrm{~g} \cdot \mathrm{mL}^{-1}\end{array}$ & $\begin{array}{l}10^{3} J_{\mathrm{e}} \\
\mathrm{Pa}^{-1} \\
\end{array}$ & $\begin{array}{c}c \\
\mathrm{~g} \cdot \mathrm{mL}^{-1}\end{array}$ & $\begin{array}{l}10^{3} J_{\mathrm{e}} \\
\mathrm{Pa}^{-1} \\
\end{array}$ & $\begin{array}{c}c \\
\mathrm{~g} \cdot \mathrm{mL}^{-1}\end{array}$ & $\begin{array}{l}10^{3} \mathrm{~J}_{\mathrm{e}} \\
\mathrm{Pa}^{-1}\end{array}$ \\
\hline 30 & $0.27_{5}$ & 0.458 & $0.26_{7}$ & 0.197 & $0.25_{2}$ & 0.308 & - & - \\
\hline 20 & 0.189 & 1.43 & $0.19_{0}$ & 0.452 & $0.17_{7}$ & 0.551 & $0.15_{3}$ & 1.89 \\
\hline 12 & $0.11_{6}$ & 2.88 & $0.12_{1}$ & 0.740 & $0.10_{8}$ & 0.956 & $0.09_{3}$ & 6.58 \\
\hline 8 & $0.07_{3}$ & 2.98 & $0.07_{3}$ & 1.04 & $0.07_{6}$ & 1.44 & $0.05_{6}$ & 6.92 \\
\hline 6 & $0.05_{8}$ & 3.43 & $0.05_{3}$ & 2.28 & $0.06_{0}$ & 2.44 & $0.04_{9}$ & 18.1 \\
\hline 3 & 0.029 & - & $0.03_{5}$ & - & $0.02_{8}$ & - & $0.02_{2}$ & - \\
\hline
\end{tabular}


Figure S12a and S12b show the difference of the $c$-dependence of the reduced viscosities using $M_{\mathrm{w}, \text { total }}$ and $M_{\mathrm{w}, \mathrm{L}}$, respectively, for D-30/240/30 solutions comparing with linear PS ones.
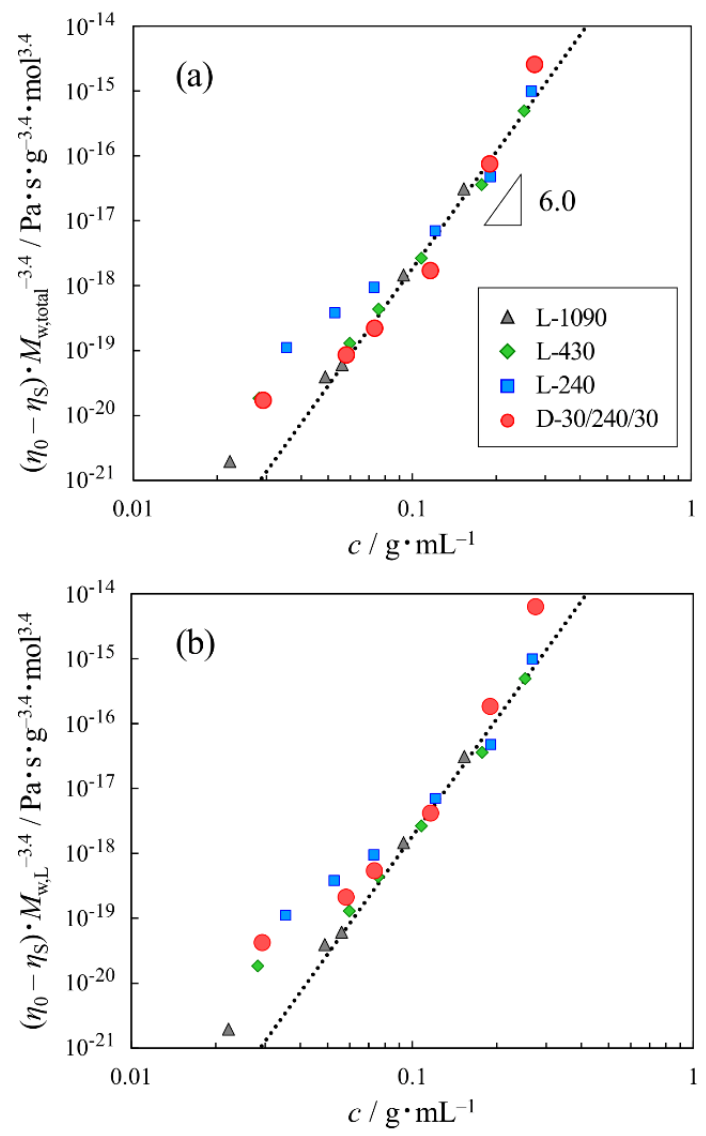

Figure S12. Concentration dependence of the viscosity (a) $\left(\eta_{0}-\eta_{\mathrm{s}}\right) / M_{\mathrm{w}, \text { total }}{ }^{3.4}$ and (b) $\left(\eta_{0}-\eta_{\mathrm{s}}\right) / M_{\mathrm{w}, \mathrm{L}}{ }^{3.4}$ for D-30/240/30 solutions in DOP at $22{ }^{\circ} \mathrm{C}$, compared with those for linear PS solutions. Dotted lines indicate the approximate straight lines for linear PS solutions with a slope of 6.0 at high $c$ regime. Note that the panel (b) is the same with Figure $6 \mathrm{~b}$ in the main text. 
Figure S13 shows the concentration dependence of the steady-state compliances $J_{\mathrm{e}}$ for D-30/240/30 solutions in DOP compared with those for linear PS ones.

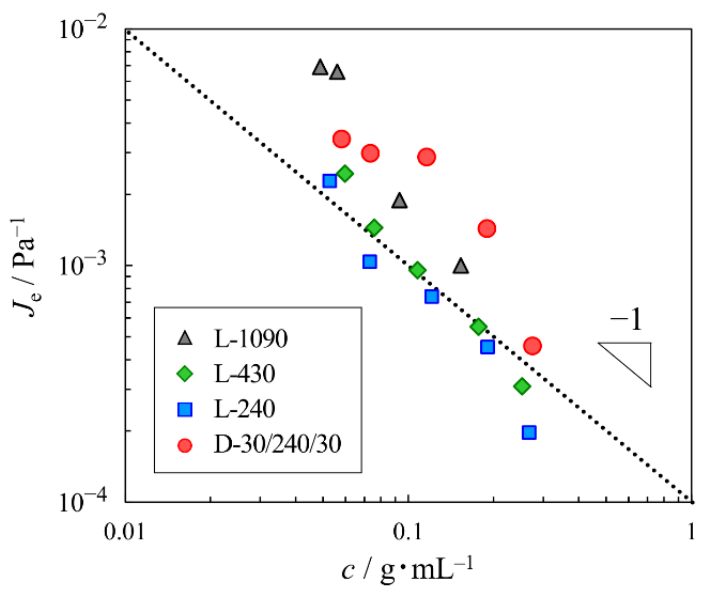

Figure S13. Concentration dependence of the steady-state compliances $J_{\mathrm{e}}$ for D-30/240/30 solutions in DOP at $22{ }^{\circ} \mathrm{C}$, compared with those for linear PS solutions. Dotted line indicates the approximate straight line for linear PS solutions with a slope of -1 .

\section{References:}

S1. Doi, Y.; Takano, A.; Matsushita, Y. Synthesis and Characterization of Dumbbell-Shaped Polystyrene. Polymer 2016, 106, 8-13.

S2. Doi, Y.; Ohta, Y.; Nakamura, M.; Takano, A.; Takahashi, Y.; Matsushita, Y. Precise Synthesis and Characterization of Tadpole-Shaped Polystyrenes with High Purity. Macromolecules 2013, 46, 1075-1081.

S3. Tobolsky, A. V.; Murakami, K. Existence of a Sharply Defined Maximum Relaxation Time for Monodispersed Polystyrene. J. Polym. Sci. 1959, 40, 443-456.

S4. Miyaki, Y.; Einaga, Y.; Fujita, H. Excluded-Volume Effects in Dilute Polymer Solutions. 7. Very High Molecular Weight Polystyrene in Benzene and Cyclohexane. Macromolecules 1978, 11, 1180-1186.

S5. Fetters, L.; Hadjichristidis, N.; Lindner, J. S.; Mays, J. W. Molecular Weight Dependence of Hydrodynamic and Thermodynamic Properties for Well-Defined Linear Polymers in Solution. J. Phys. Chem. Ref. Data 1994, 23, 619640 . 